\title{
Down the Wrong Way: An Important Complication of Nasogastric Tube Placement
}

\author{
Aneurin Moorthy
}

\begin{abstract}
Placement of a nasogastric tube is a very common procedure carried out by doctors and nurses on both medical and surgical wards. Indications include for feeding purpose to meet the patient's nutrition requirement and a therapeutic use to decompress a distended bowl in an acute small/large bowl obstruction. Insertion of a nasogastric tube into the pulmonary tree is a well-recognized complication that can possibly cause a pneumothorax or pulmonary aspiration if feeding was commenced without checking its position before use. This case report describes pneumothorax in a 65 -year-old patient following insertion of a nasogastric tube. It also highlights the management of pneumothorax causes by intra-pleural insertion of a nasogastric tube and patients who are susceptible to nasogastric tube complications.
\end{abstract}

Keywords: Nasogastric tube; Pneumothorax; Complications; Pleural penetration

\section{Introduction}

The insertion of a nasogastric tube is a common procedure in medical and surgical wards. Indications for nasogastric tube insertion include gastric decompression for the management of bowel obstruction, enteral nutrition feeding and delivery of medicines in patients with compromised airways. This case report describes/reminds us of an uncommon but serious complication following insertion of a fine bore nasogastric tube. It also illustrates key symptoms and signs of pneumothorax following bronchial insertion of a nasogastric tube and its management.

\section{Case Report}

A 65-year-old woman was admitted to a major teaching hospi-

Manuscript accepted for publication October 22, 2014

Department of Gastroenterology, St. James's Hospital, James's Street, Dublin 8, Co. Dublin, Ireland. Email: Aneurin.moorthy@gmail.com

doi: http://dx.doi.org/10.14740/jmc1977w tal in Co. Dublin, Ireland with a 1-week history of moderatesevere epigastric pain exacerbated by food and a weight loss of $4 \mathrm{~kg}$. She admitted taking ibuprofen regularly to alleviate the pain. She has a background history of severe vascular dementia, CVA and dyslipidemia. Endoscopy examination revealed a non-complicated gastric ulcer. She was treated conservatively as an inpatient. She was not meeting her nutrition requirements and consequently a fine bore nasogastric tube was inserted for the purpose of enteral feeding under the guidance of the dietician. A routine chest X-ray was ordered for confirmation of position of the nasogastric tube prior to feeding. One hour post insertion of the nasogastric tube, the patient began to desaturate to $84 \%$ on room air, became tachypnoeic, tachycardic, apnoeic episodes noted by the nursing staff and increasingly agitated. Auscultation of the chest revealed decreased breath sounds/air entry over the entire right lung. Review of the chest $\mathrm{X}$-ray illustrated the nasogastric tube passing into the right

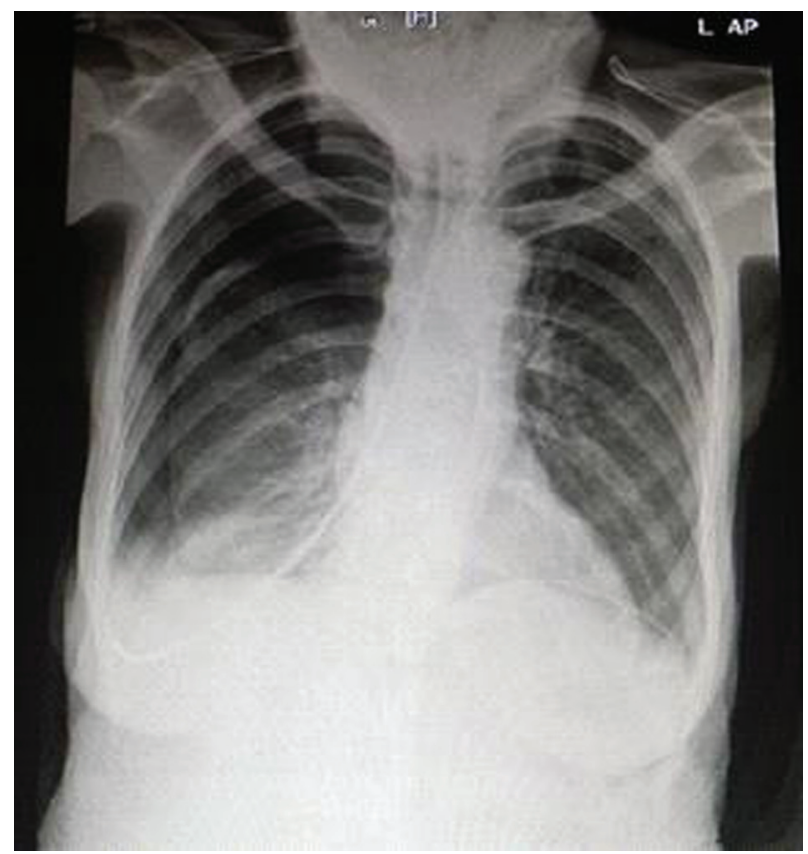

Figure 1. Advancement of the nasogastric tube into the right main bronchus and piercing the right pleura causing a $50 \%$ pneumothorax. Note the presence of the tip of the nasogastric tube in the right pleural space. 


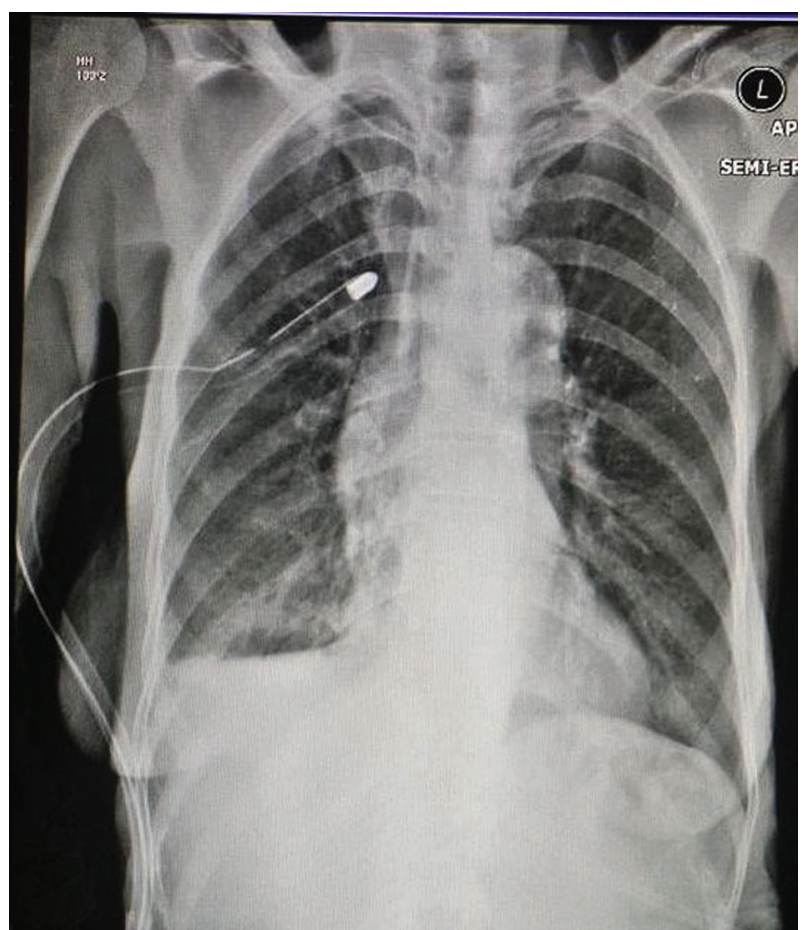

Figure 2. Re-expansion of the right lung $24 \mathrm{~h}$ post chest drain insertion. Note the presence of the chest drain and residual pneumomediastiunum

main bronchus, into the lower lobe and extending into the plural space causing a $50 \%$ pneumothorax (Fig. 1). An urgent cardiothoracic consultation was made and a 20-Fr intercostal chest drain with an underwater seal was inserted for the treatment of the pneumothorax.

Twenty-four hours later, a repeat chest X-ray showed complete expansion of the right lung with some residual pneumomediastinum (Fig. 2) and the patient had clinically improved. The chest drain was subsequently removed. Serial chest Xrays post the iatrogenic event showed persistent pneumomediastinum. With concern that this could be a sign indicating a pulmonary tree tear or esophageal tear/rupture, a computed tomography (CT) scan of the thorax was performed. Perforation and dissection of the esophagus have been reported in the literature [1]. CT of thorax did not show any evidence of esophageal or tracheobronchial tear.

\section{Discussion}

Placement of a nasogastric tube into the pulmonary tree is a well-known complication [2, 3]. Its incidence ranges from $0.3 \%$ to $15 \%$ [2]. Fine nasogastric tubes are used for enteral feeding. A metal stylet is usually needed to increase rigidity to allow easier insertion. However, this increases the risk of penetrating the bronchus, small airways or the pleura [2]. The gold standard to ensure proper positioning of the nasogastric tube post insertion is visualizing the tip of the tube below the diaphragm on a chest X-ray [2].

An important clinical lesson/experience from this case is to identify patients that are at risk of nasogastric tube complications. Risk factors include recent nasal fracture, basal skull fractures, patients with compromised airways, heavily sedated/ confused patients, elderly and patients with neurological impairment that decreases their swallow/gag reflex [2, 4-6]. The patient from this case report had two of these risk factors, elderly and severe vascular dementia which in turn is a risk factor for impaired gag reflex [7]. Another important lesson to be gained from this case is the importance of high index of suspicion of tube malposition by doctors and nurses in patients with acute respiratory distress following tube placement. This case report illustrates key signs and symptoms of respiratory distress that should be identified that suggest tube misplacement.

On review of the literature, there are different ways to prevent pneumothorax in high risk patients. One method describes the passage of the nasogastric tube up to $30-35 \mathrm{~cm}$ and followed by a chest X-ray assessment of its position before further advancement of the tube. At $30-35 \mathrm{~cm}$ advancement of the tube, any abnormal curvature of the nasogastric tube can be identified, thus halting further advancement of the tube. If the tube is centered, it is highly likely to be positioned in the esophagus and can be further advanced for optimum length with repeat chest X-ray to confirm its position $[4,5]$. Another method describes a simple bedside test. The tube is advanced again to $30 \mathrm{~cm}$ and capnography is used to detect high levels of carbon dioxide. High levels indicate the presence of the tube into pulmonary tree and therefore the nasogastric tube should be withdrawn rather advanced to prevent a potential subsequent pneumothorax [4].

In conclusion, doctors and nurses should carefully identify patients at high risk of complication of nasogastric tube insertion prior to tube placement, and be aware of important signs and symptoms that suggest tube misplacement and its management.

\section{Grant}

None.

\section{Conflict of Interest}

None.

\section{References}

1. McWey RE, Curry NS, Schabel SI, Reines HD. Complications of nasoenteric feeding tubes. Am J Surg. 1988;155(2):253-257.

2. Smith LN, Park MF. Case Report and Review- Nasogastric tube complications. Crit Care\& shock. 2012;15(2):3642.

3. O'Neil R, Krishnananthan R. Intrapleural nasogastric tube insertion. Australas Radiol. 2004;48(2):139-141.

4. Pillai JB, Vegas A, Brister S. Thoracic complications of nasogastric tube: review of safe practice. Interact Cardio- 
vasc Thorac Surg. 2005;4(5):429-433.

5. Lo JO, Wu V, Reh D, Nadig S, Wax MK. Diagnosis and management of a misplaced nasogastric tube into the pulmonary pleura. Arch Otolaryngol Head Neck Surg. 2008;134(5):547-550.

6. Gregory JA, Turner PT, Reynolds AF. A complication of nasogastric intubation: intracranial penetration. J Trauma. 1978;18(12):823-824.

7. Stephanie KD. Neurological disorders affecting oral, pharyngeal swallowing. GI motility online [Internet]. 2006 May [cited: 2014 September] Available from: http:// www.nature.com/gimo/contents/pt1/full/gimo34.html. 